\title{
New Circumstellar Sources of PO and PN: The Increasing Role of Phosphorus Chemistry in Oxygen-rich Stars
}

\author{
L. M. Ziurys ${ }^{1,2}$, D. R. Schmidt ${ }^{2}$, and J. J. Bernal ${ }^{1}$ \\ ${ }^{1}$ Department of Chemistry and Biochemistry, University of Arizona, 1305 E. 4th Street, Tucson, AZ 85719, USA; halfend@email.arizona.edu \\ ${ }^{2}$ Department of Astronomy, Arizona Radio Observatory, University of Arizona, 933 N. Cherry Avenue, Tucson, AZ 85721, USA \\ Received 2017 September 1; revised 2018 January 26; accepted 2018 February 12; published 2018 April 5
}

\begin{abstract}
PO and PN have been newly identified in several oxygen-rich circumstellar envelopes, using the Submillimeter Telescope of the Arizona Radio Observatory. The $J=5 \rightarrow 4$ and $J=6 \rightarrow 5$ transitions of PN near 235 and $282 \mathrm{GHz}$, and the lambda doublets originating in the $J=5.5 \rightarrow 4.5$ and $J=6.5 \rightarrow 5.5$ lines of PO at 240 and $284 \mathrm{GHz}$, have been detected toward the shells of asymptotic giant branch (AGB) stars TX Cam and R Cas. A similar set of lines has been observed toward the supergiant NML Cyg, and new transitions of these two molecules were also measured toward the AGB star IK Tau. Along with the previous data from VY Canis Majoris (VY CMa), these spectral lines were analyzed using the non-local thermodynamic equilibrium (non-LTE) circumstellar modeling code, ESCAPADE. For the AGB stars, peak abundances found for PN and PO were $f \sim(1-2) \times 10^{-8}$ and $(0.5-1) \times 10^{-7}$, respectively, while those for the supergiants were $f(\mathrm{PN}) \sim(0.3-0.7) \times 10^{-8}$ and $f(\mathrm{PO}) \sim(5-7) \times 10^{-8}$. PN was well modeled with a spherical radial distribution, suggesting formation near the stellar photosphere, perhaps enhanced by shocks. PO was best reproduced by a shell model, indicating a photochemical origin, except for VY CMa. Overall, the abundance of PO is a factor of 5-20 greater than that of PN. This study suggests that phosphorus-bearing molecules are common in O-rich envelopes, and that a significant amount of phosphorus $(>20 \%)$ remains in the gas phase.
\end{abstract}

Key words: astrochemistry - circumstellar matter - ISM: molecules - methods: observational - stars: abundances submillimeter: stars

\section{Introduction}

The origin and evolution of interstellar phosphorous remains something of an enigma. Phosphorus is clearly a key biogenic element, playing critical roles in replication, metabolism, and cellular structure (e.g., Maciá 2005). However, unlike the other biogenic elements $\mathrm{H}, \mathrm{C}, \mathrm{O}$, and $\mathrm{N}$, it has a relatively low cosmic abundance of $3 \times 10^{-7}$ (e.g., Asplund et al. 2009) and has only one stable isotope, ${ }^{31} \mathrm{P}$. Furthermore, its origin in nucleosynthesis is not well understood. Phosphorus is thought to be mainly produced by core-collapse in supernovae $(\mathrm{SNe})$, with a small possible contribution from SNe Ia (e.g., Cescutti et al. 2012). During O- and Ne-shell burning, neutron capture on ${ }^{29} \mathrm{Si}$ and ${ }^{30} \mathrm{Si}$ creates ${ }^{31} \mathrm{P}$, although a large fraction of the phosphorus can be subsequently destroyed by (p, $\alpha$ ) reactions, leading to ${ }^{28} \mathrm{Si}$. However, confirming this shell-burning origin has been difficult, as the element has been elusive. There are no optical transitions of phosphorus that can be readily observed in ordinary $\mathrm{F}, \mathrm{G}$, and $\mathrm{K}$ stars, thus severely limiting the determination of stellar yields (e.g., Jacobsen et al. 2014). PI lines in the infrared and ultraviolet have been observed in stars as an alternative, albeit with some difficulty (Caffau et al. 2011; Jacobsen et al. 2014). Such studies have led to the conclusion that the yields from massive stars are currently insufficient to account for the observed abundances of phosphorus (e.g., Cescutti et al. 2012).

In the interstellar medium (ISM), the element has been studied via its PI, PII, and PIII lines, observed in absorption in the ultraviolet toward diffuse clouds, where it appears to be slightly depleted or undepleted (e.g., Savage \& Sembach 1996; Lebouteiller et al. 2006). Recently, phosphorus has been observed in the near-infrared (-IR) toward the supernova remnant $\mathrm{Cas} \mathrm{A}$; in this object, the abundance was found to be greatly enhanced relative to the general Milky Way. This result has been interpreted as evidence for its production in SNe. In circumstellar envelopes of asymptotic giant branch (AGB) stars, thermodynamic calculations predict that phosphorus will condense into schreibersite grains $(\mathrm{Fe}, \mathrm{Ni})_{3} \mathrm{P}$ (e.g., Lodders \& Fegley 1999). Yet, schreibersite has yet to be identified in presolar grains, which are thought to originate in stellar ejecta (McSween \& Huss 2010), although it is common in iron meteorites (e.g., Pasek \& Lauretta 2005).

Millimeter-wave molecular-line observations have proven to provide another avenue by which to probe the phosphorus abundance in the ISM. The first detection of this element in molecular form was as PN, which was observed in 1987 toward several molecular clouds (Ziurys 1987). PN has subsequently been identified toward other dense clouds and in their massive cores (e.g., Turner et al. 1990; Fontani et al. 2016). Other P-containing compounds, however, have been elusive in molecular clouds, although PO has recently been observed in L1157 (Lefloch et al. 2016). Circumstellar envelopes, on the other hand, have exhibited a far richer phosphorus inventory. Toward the shell of the famous C-rich star IRC +10216 , PN, CP, CCP, HCP, and $\mathrm{PH}_{3}$ have been detected (e.g., Agúndez et al. 2007, 2008, 2014; Halfen et al. 2008; Milam et al. 2008; Tenenbaum \& Ziurys 2008). Several of these compounds have also been identified in the C-rich shells of evolved stars CRL 618 and CRL 2688 (e.g., Milam et al. 2008). Perhaps the most striking phosphorus-related detection has been the species PO, which was discovered in the envelope of the oxygen-rich supergiant VY Canis Majoris, or VY CMa (Tenenbaum et al. 2007) - the first identification of a P-O bond in space. Along with PO, PN has also been found in this object. More recently, both PO and PN have been identified in the O-rich AGB star IK Tauri (IK Tau) by De Beck et al. (2013). These results clearly 
Table 1

Properties of Observed Stars

\begin{tabular}{|c|c|c|c|c|c|c|}
\hline Source & $\alpha(\mathrm{J} 2000.0)$ & $\delta(\mathrm{J} 2000.0)$ & $\begin{array}{c}R_{\star} \\
(\mathrm{cm})\end{array}$ & $\begin{array}{c}T_{\star} \\
(\mathrm{K})\end{array}$ & $\begin{array}{l}\text { Distance } \\
\text { (pc) }\end{array}$ & $\begin{array}{l}\text { Mass-loss Rate } \\
\left(M_{\odot} \mathrm{yr}^{-1}\right)\end{array}$ \\
\hline TX Cam & $05^{\mathrm{h}} 00^{\mathrm{m}} 50^{\mathrm{s}} \cdot 4$ & $56^{\circ} 10^{\prime} 53^{\prime \prime}$ & $2.0 \times 10^{13 \mathrm{a}}$ & $2600^{\mathrm{a}}$ & $390^{\mathrm{a}}$ & $4 \times 10^{-6 \mathrm{~b}}$ \\
\hline IK Tau & $03^{\mathrm{h}} 53^{\mathrm{m}} 28^{\mathrm{s}} .7$ & $11^{\circ} 24^{\prime} 23^{\prime \prime}$ & $2.1 \times 10^{13 c}$ & $2200^{\mathrm{d}}$ & $250^{\mathrm{e}}$ & $5 \times 10^{-6 b}$ \\
\hline VY CMa & $07^{\mathrm{h}} 22^{\mathrm{m}} 58^{\mathrm{s}} \cdot 3$ & $-25^{\circ} 46^{\prime} 03^{\prime \prime}$ & $1.4 \times 10^{14 \mathrm{i}}$ & $2800^{\mathrm{i}}$ & $1140^{\mathrm{j}}$ & $1.85 \times 10^{-4 \mathrm{i}}$ \\
\hline NML Cyg & $20^{\mathrm{h}} 46^{\mathrm{m}} 25^{\mathrm{s}} .5$ & $40^{\circ} 06^{\prime} 59^{\prime \prime}$ & $2.6 \times 10^{-14 a}$ & $2500^{\mathrm{k}}$ & $1740^{1}$ & $2 \times 10^{-4 \mathrm{~m}}$ \\
\hline
\end{tabular}

Notes.

${ }^{\text {a }}$ Ziurys et al. (2009).

${ }^{\mathrm{b}}$ Maercker et al. (2016).

${ }^{c}$ Duari et al. (1999).

${ }^{\mathrm{d}}$ Decin et al. (2010).

e Olofsson et al. (1998).

${ }^{\mathrm{f}}$ Markwick \& Millar (2000).

g Maercker et al. (2008).

${ }^{\mathrm{h}}$ De Beck et al. (2010).

${ }^{\mathrm{i}}$ Matsuura et al. (2014).

${ }^{\mathrm{j}}$ Choi et al. (2008).

${ }^{\mathrm{k}}$ Monnier et al. (1997).

${ }^{1}$ Massey \& Thompson (1991).

m Justtanont et al. (1996).

demonstrate that circumstellar envelopes have a complex phosphorus chemistry.

In order to further probe the presence of phosphorus in O-rich stars, we have conducted new observations of both $\mathrm{PO}$ and $\mathrm{PN}$ at $1 \mathrm{~mm}$ toward the envelopes of three oxygen-rich AGB stars, R Cas, TX Cam, and IK Tau, as well as the O-rich supergiant NML Cyg, using the Submillimeter Telescope (SMT) of the Arizona Radio Observatory (ARO). PN and PO were detected for the first time toward R Cas, TX Cam, and NML Cyg. Our measurements toward IK Tau were conducted before the De Beck et al. (2013) work appeared, but concern different transitions, and thus are complementary. These new data have been modeled with the nonLTE circumstellar radiative transfer code ESCAPADE (Adande et al. 2013), along with spectra obtained previously for PO and PN toward the supergiant star VY CMa in the ARO $1 \mathrm{~mm}$ spectral-line survey of Tenenbaum et al. (2010). From this analysis, abundances and radial distributions were determined. Here we present our observations and results, and discuss their implications for phosphorus chemistry in O-rich circumstellar gas.

\section{Observations}

The measurements were conducted during the period 2010 November to 2016 December using the ARO SMT on Mount Graham, Arizona. A dual polarization, $1 \mathrm{~mm}$ receiver comprised of the Atacama Large Millimeter/Submillimeter Array (ALMA) Band 6, sideband-separating mixers was utilized. Image rejection was typically $\geqslant 17 \mathrm{db}$, which was inherent in the mixer architecture. The intensity scale at the SMT is $T_{\mathrm{A}}{ }^{*}$, the standard chopper-wheel antenna temperature, and has an uncertainty of $15 \%$. The radiation temperature is then defined as $T_{\mathrm{R}}=T_{\mathrm{A}}^{*} / \eta_{\mathrm{b}}$, where $\eta_{\mathrm{b}}$ is the main beam efficiency. Typical system temperatures were in the range $200-400 \mathrm{~K}$, single sideband (SSB), and the rms noise levels achieved were 0.1-1 mK (see Table 3). The backends employed were 2048channel, $1 \mathrm{MHz}$ resolution filter banks operated in parallel mode $(2 \times 1024$ channels $)$.

Observations were conducted in a beam-switching mode with a subreflector throw of $\pm 2^{\prime}$. Telescope pointing and focus
Table 2

Rest Frequencies and Telescope Parameters

\begin{tabular}{llccc}
\hline \hline Molecule & Transition & Frequency $(\mathrm{MHz})$ & $\eta_{\mathrm{b}}$ & $\theta_{\mathrm{b}}\left({ }^{\prime \prime}\right)$ \\
\hline $\mathrm{PN}\left(\mathrm{X}^{1} \Sigma\right)$ & $J=5 \rightarrow 4$ & 234935.7 & 0.76 & 32 \\
& $J=6 \rightarrow 5$ & 281914.1 & 0.72 & 26 \\
$\mathrm{PO}\left(\mathrm{X}^{2} \Pi_{\mathrm{r}}\right): \Omega=1 / 2$ & $J=5.5 \rightarrow 4.5$ & & & \\
& $e: F=6 \rightarrow 5$ & $239949.0^{\mathrm{a}}$ & 0.76 & 31 \\
& $F=5 \rightarrow 4$ & $239958.1^{\mathrm{a}}$ & & \\
& $f: F=6 \rightarrow 5$ & $240141.1^{\mathrm{a}}$ & 0.76 & 31 \\
& $F=5 \rightarrow 4$ & $240152.5^{\mathrm{a}}$ & & \\
& $J=6.5 \rightarrow 5.5$ & & & \\
& $e: F=7 \rightarrow 6$ & $283586.8^{\mathrm{a}}$ & 0.72 & 26 \\
$F=6 \rightarrow 5$ & $283593.2^{\mathrm{a}}$ & & \\
& $f: F=7 \rightarrow 6$ & $283777.6^{\mathrm{a}}$ & 0.72 & 26 \\
$F=6 \rightarrow 5$ & $283785.4^{\mathrm{a}}$ & & \\
\hline
\end{tabular}

Note.

${ }^{a}$ Hyperfine lines usually blended in the data; see Table 3.

were monitored on strong continuum sources. The pointing accuracy is $\pm 3^{\prime \prime}$. For all of the measurements, the local oscillator was shifted by $10 \mathrm{MHz}$ to check for image contamination. Sources and their coordinates are listed in Table 1; rest frequencies, beam sizes, and telescope efficiencies are summarized in Table 2.

\section{Results}

PN and PO were detected in all three O-rich AGB envelopes, IK Tau, R Cas, and TX Cam, as well as the supergiant NML Cyg. Note that both species had been identified previously in IK Tau by De Beck et al. (2013). Spectra for the AGB shells are presented in Figure 1. Here the $J=5 \rightarrow 4$ and $J=6 \rightarrow 5$ transitions of PN are shown (left), which consist of single lines, as indicated by arrows underneath the data; the $J=5.5 \rightarrow 4.5$ and $J=6.5 \rightarrow 5.5$ transitions of PO (right) are displayed as well, each of which consist of lambda doublets, labeled by $e$ and $f$, and indicated by arrows. The $J=5 \rightarrow 4$ line of PN and the $J=5.5 \rightarrow 4.5$ 
(a)

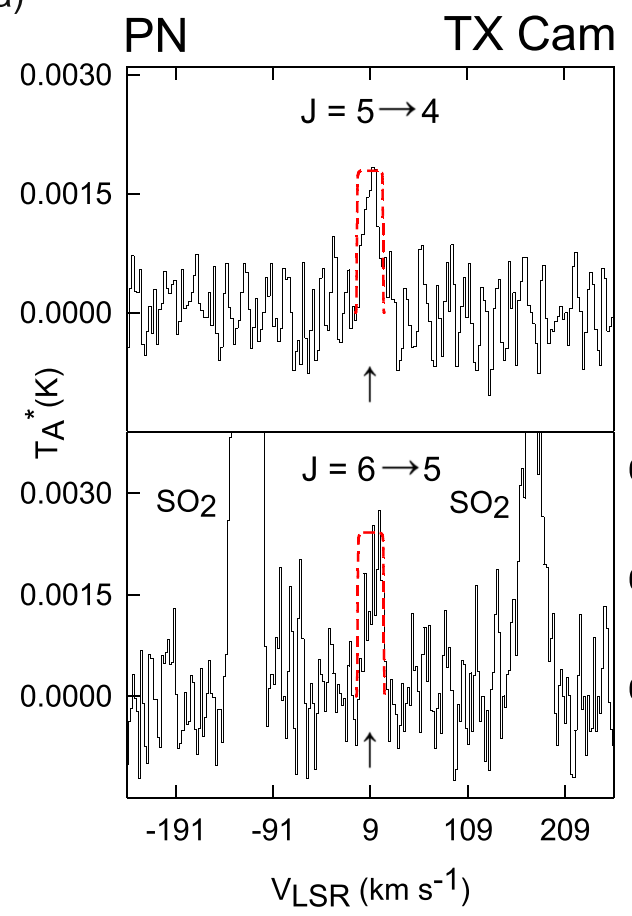

(b)

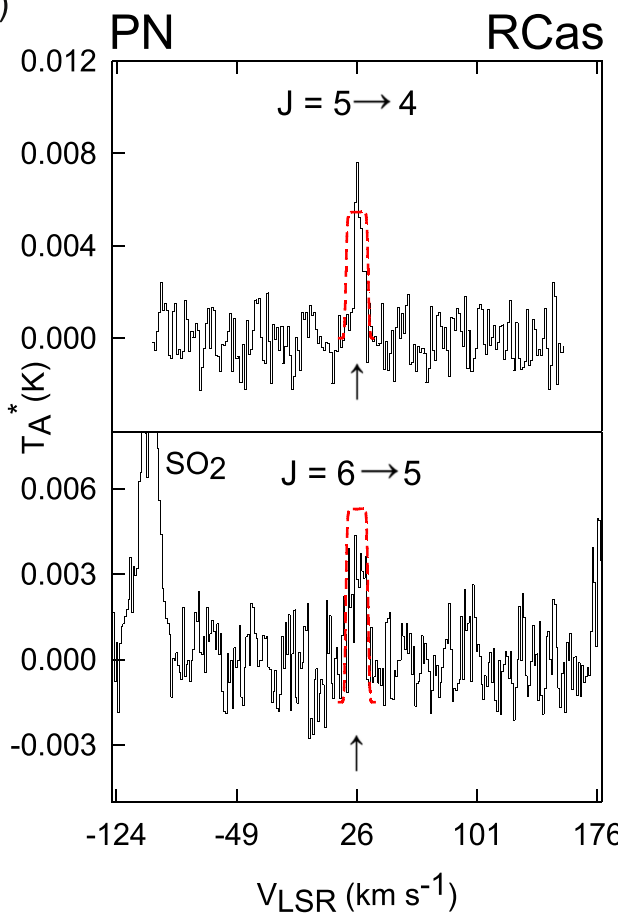

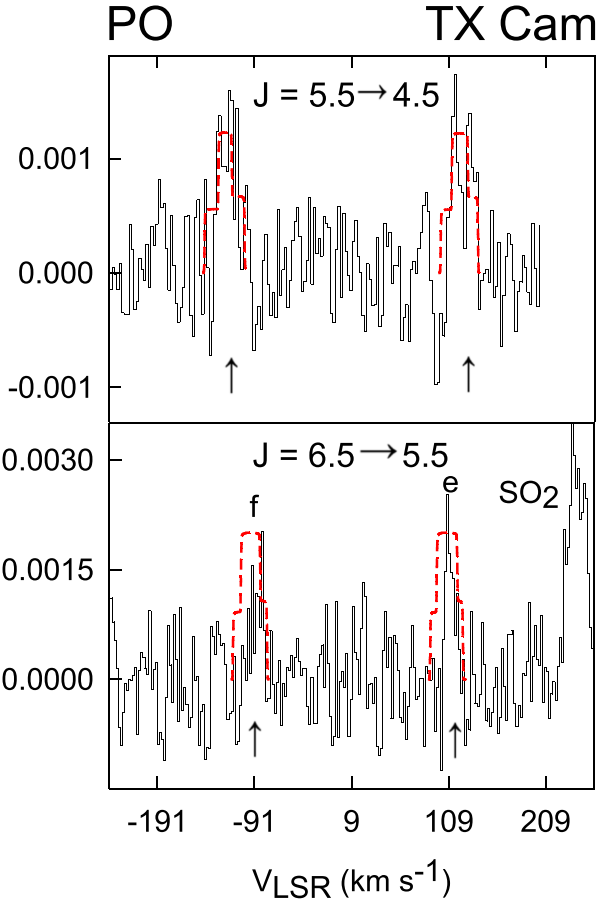

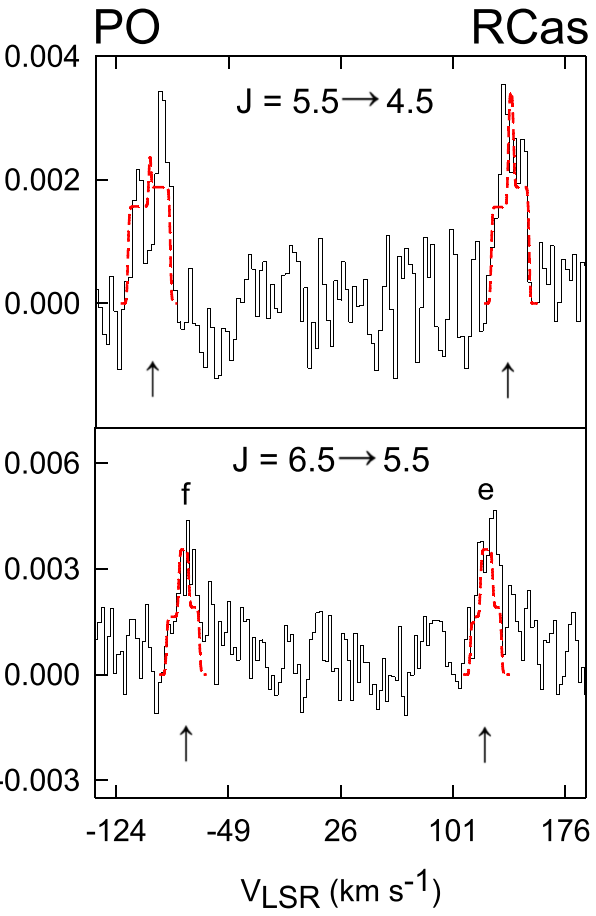

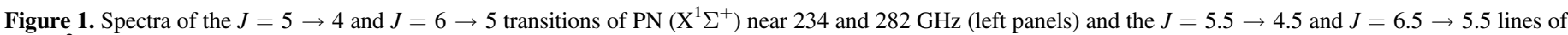

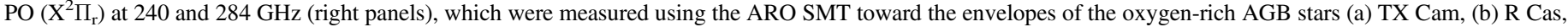

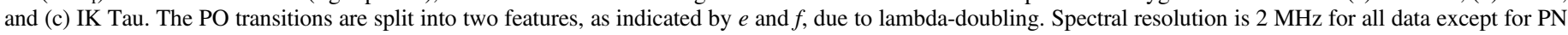

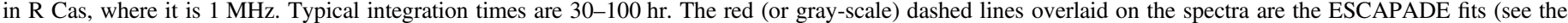
text). Other spectral lines are visible in the data arising from $\mathrm{SO}_{2}$. These spectra verify the identification of PN and PO in TX Cam and R Cas.

doublets of PO had not been previously observed in IK Tau (Figure 1(c)). These data clearly confirm the presence of PO and PN in two new AGB stars: TX Cam and R Cas (Figures 1(a) and (b)). The red line overlay on all the spectra are the model fits, which will be discussed later.

Figure 2 presents the PO and PN spectra observed toward the envelopes of the two supergiant stars, NML Cyg and VY CMa.
Again, the red overlays are the model fits. Only the $J=6 \rightarrow 5$ transition was detected toward NML Cyg, as shown in Figure 2(a) (left panels), but the lambda doublets of the $J=5.5 \rightarrow 4.5$ and $J=6.5 \rightarrow 5.5$ transitions of $\mathrm{PO}$ were both observed (right panels). Phosphorus-bearing molecules had not been identified previously in this source. Both PO and PN were discovered several years ago in VY CMa by Tenenbaum et al. (2007) and 
(c)
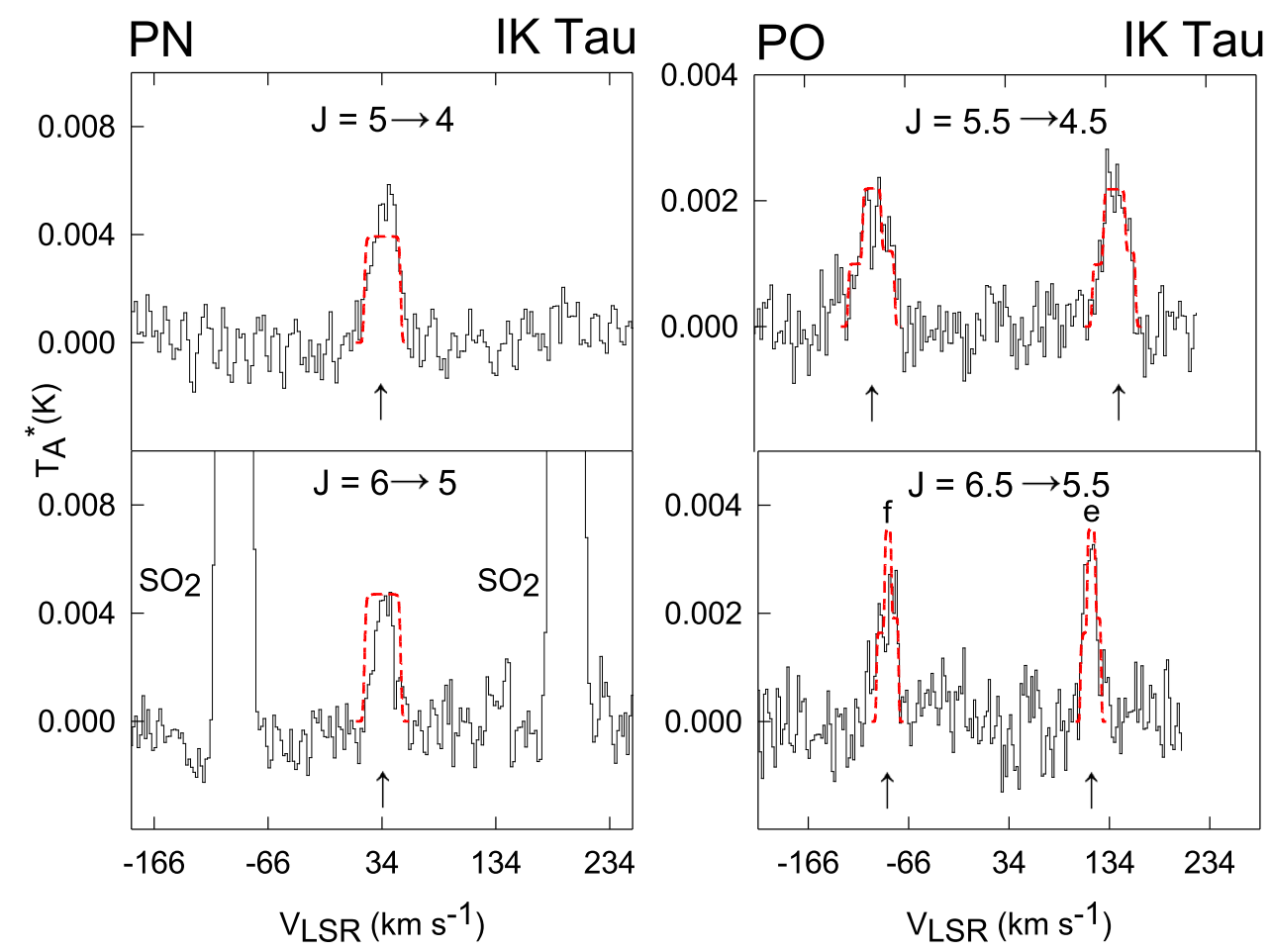

Figure 1. (Continued.)

(a)

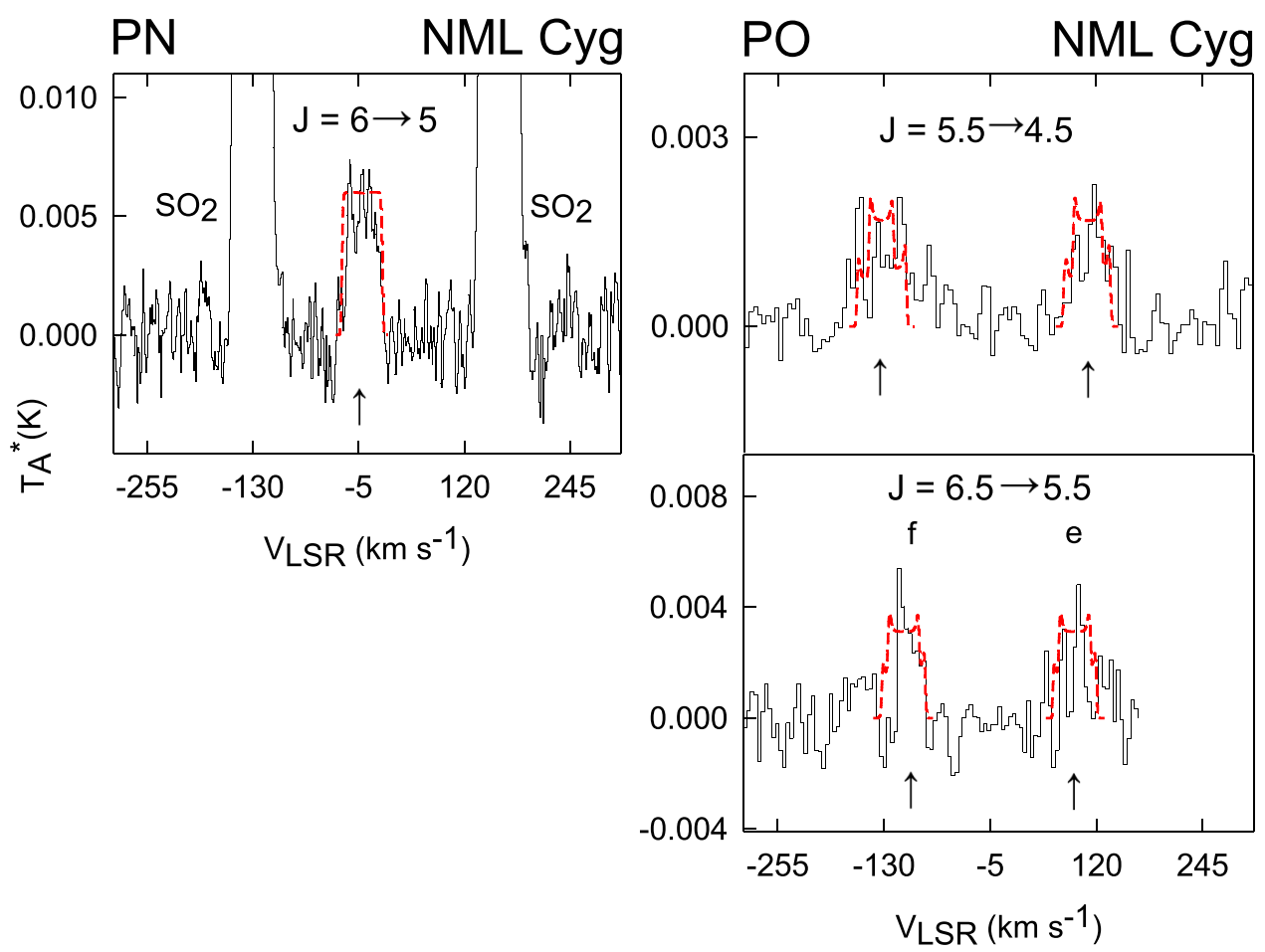

Figure 2. Spectra of the $J=5 \rightarrow 4$ and $J=6 \rightarrow 5$ transitions of $\mathrm{PN}\left(\mathrm{X}^{1} \Sigma^{+}\right)$(left panels) and the lambda doublets of the $J=5.5 \rightarrow 4.5$ and $J=6.5 \rightarrow 5.5$ lines of PO $\left(\mathrm{X}^{2} \Pi_{\mathrm{r}}\right)$, indicated by $e$ and $f$ (right panels), which were measured using the ARO SMT toward the envelopes of the oxygen-rich supergiants (a) NML Cyg and (b) VY CMa (from Tenenbaum et al. 2007; Ziurys et al. 2007; Milam et al. 2008). Also included for VY CMa is the $J=3 \rightarrow 2$ line of PN near $140 \mathrm{GHz}$, observed with the ARO $12 \mathrm{~m}$ (see Milam et al. 2008). Only one transition of PN was observed toward NML Cyg ( $J=6 \rightarrow 5$ line). Spectral resolution is $2 \mathrm{MHz}$ for the VY CMa data, except for the PN $1 \mathrm{~mm}$ transitions, where it is $1 \mathrm{MHz}$. For NML Cyg, spectral resolution is $1 \mathrm{MHz}$ and $4 \mathrm{MHz}$, respectively, for PN and PO. Typical integration times are 10-70 hr. The red (or gray-scale) dashed lines overlaid on the spectra are the ESCAPADE fits (see the text). $\mathrm{SO}_{2}$ and $\mathrm{SiS}$ lines also appear in some of the spectra. 
(b)
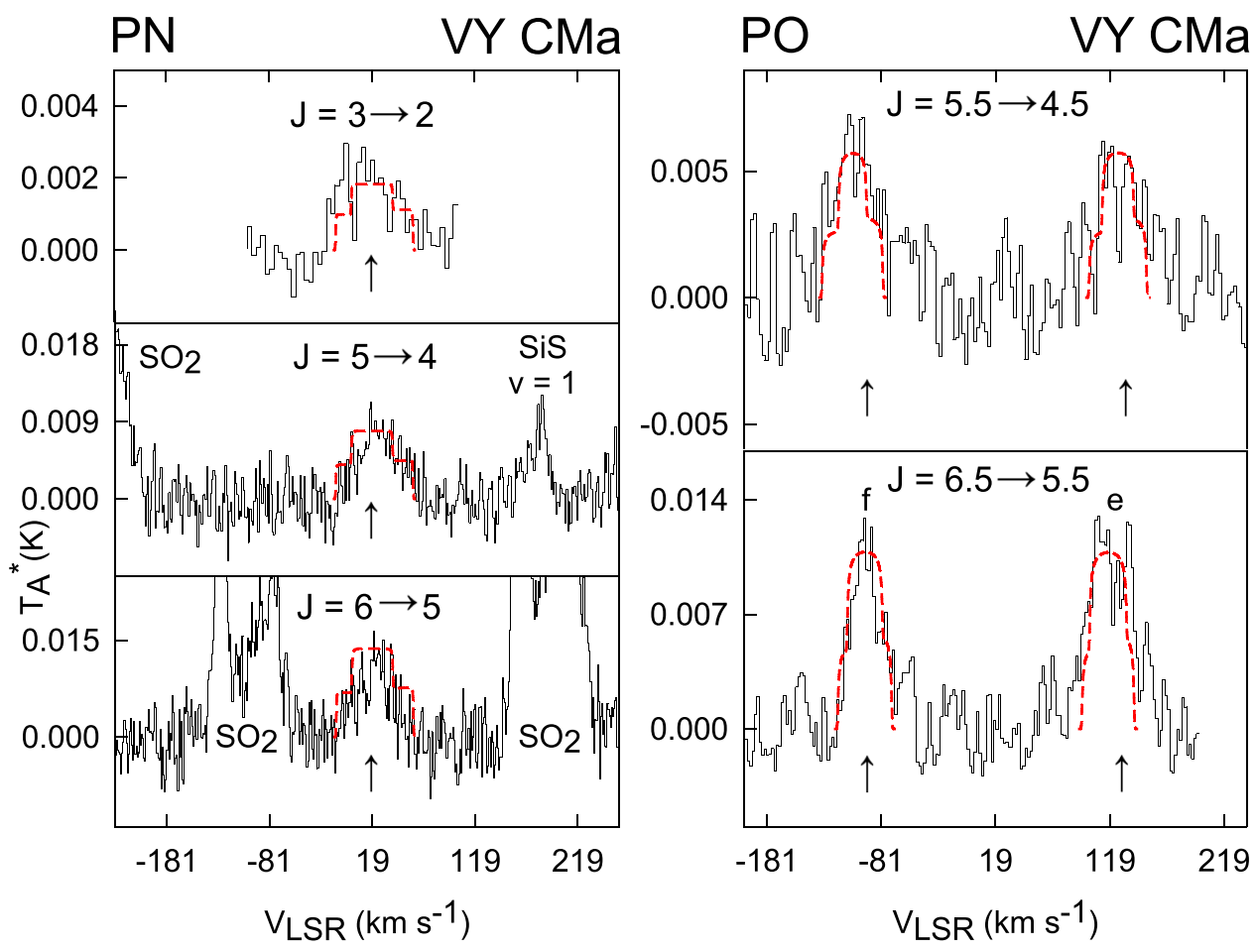

Figure 2. (Continued.)

Ziurys et al. (2007), but are shown here because of new modeling results. The spectra for VY CMa were taken from the Tenenbaum et al. (2010) spectral survey. As was also found for the AGB stars, $\mathrm{SO}_{2}$ lines are common in the spectra.

Line parameters for PO and PN for TX Cam, R Cas, IK Tau, NML Cyg, and VY CMa are given in Table 3. This table lists the line intensities (in $\left.T_{\mathrm{A}}{ }^{*}\right), \mathrm{LSR}$ velocity, and FWHM $\left(\Delta V_{1 / 2}\right)$ of all observed features. Note that with one exception, the two phosphorus hyperfine components that compose each lambda doublet are blended, and the line parameters are for the combined feature. Parameters for VY CMa are from Tenenbaum et al. (2007, 2010).

\section{Analysis}

In order to obtain abundances and envelope radial distributions, the spectra were modeled with the non-LTE radiative transfer code ESCAPADE (Adande et al. 2013). The code employs the Sobolev approximation, assuming a uniform expansion velocity, and can be used to model either spherically symmetric or directional, conical outflows. In the spherical case, the outflow is depicted by finite element shells in which the equations of statistical equilibrium are solved. Collimated outflows are represented as conical sections of the sphere, with a specified orientation (position angle) and cone width (vertex angle; see Adande et al. 2013). Rotational levels originating in the ground and first vibrationally excited states are modeled, considering excitation by collisions, as well as by infrared radiation from dust. Once the level populations are determined, the equation of radiative transfer is directly integrated at each shell radius across the given outflow. The emergent radiation is convolved with the telescope beam, yielding the beam diluted brightness temperature, as observed. Any molecule can be modeled with ESCAPADE, independent of geometry or the molecular term.
Further details are given in Adande et al. (2013), and the complete code can be found in Adande (2013).

For PN, the lowest 60 rotational levels of both the ground and first excited vibrational states were considered. Note that $\Delta E=1904 \mathrm{~K}$ for the $v=1$ level. The first 40 energy levels (up to $J=9.5, F=9.0$ ) of the ground vibrational state and the first excited vibrational state $(\Delta E=1756 \mathrm{~K})$ for the $\Omega=1 / 2$ ladder of $\mathrm{PO}$ were modeled. Collision rates with $\mathrm{H}_{2}$ were obtained from the BASECOL database (Dubernet et al. 2013). Those for phosphorus nitride were scaled from the $\mathrm{PN}-\mathrm{He}$ rates of Tobola et al. (2007) and covered the temperature range of $10-300 \mathrm{~K}$ in increments of $10 \mathrm{~K}$. Rates for temperatures above $300 \mathrm{~K}$ were extrapolated from those in the $100-300 \mathrm{~K}$ range. Rates involving PO are not yet available in the literature, so estimates were obtained by scaling from those of the NO-He system, as measured by Kłos et al. (2008). The NO rates were used as PO and NO are isovalent and possess ${ }^{2} \Pi$ ground states. The ratio of the reduced masses was used to scale the NO-He system to the $\mathrm{NO}-\mathrm{H}_{2}$ system and then similarly to the $\mathrm{PO}-\mathrm{H}_{2}$ system. In this case, the temperature range extended from $10 \mathrm{~K}$ to $500 \mathrm{~K}$ in increments of $10 \mathrm{~K}$; again, for higher temperatures, the rates were extrapolated.

Both gas kinetic temperature and density profiles are needed in ESCAPADE to model collisional excitation. The dust is assumed to radiate as a blackbody at a given $T_{\text {dust }}$ for the infrared excitation (Adande et al. 2013). The gas kinetic temperature radial profiles are modeled as the power law (e.g., see Ziurys et al. 2009):

$$
T_{\text {kin }}(r)=T_{\text {profile }}\left(\frac{R_{\text {profile }}}{r}\right)^{\gamma} .
$$

Here $T_{\text {profile }}$ is the gas kinetic temperature at distance $R_{\text {profile }}$ from the star. The values for $T_{\text {profile }}, R_{\text {profile, and } \gamma \text { for the }}$ 
Table 3

Line Parameters of Observed Transitions of $\mathrm{PN}$ and $\mathrm{PO}^{\mathrm{a}}$

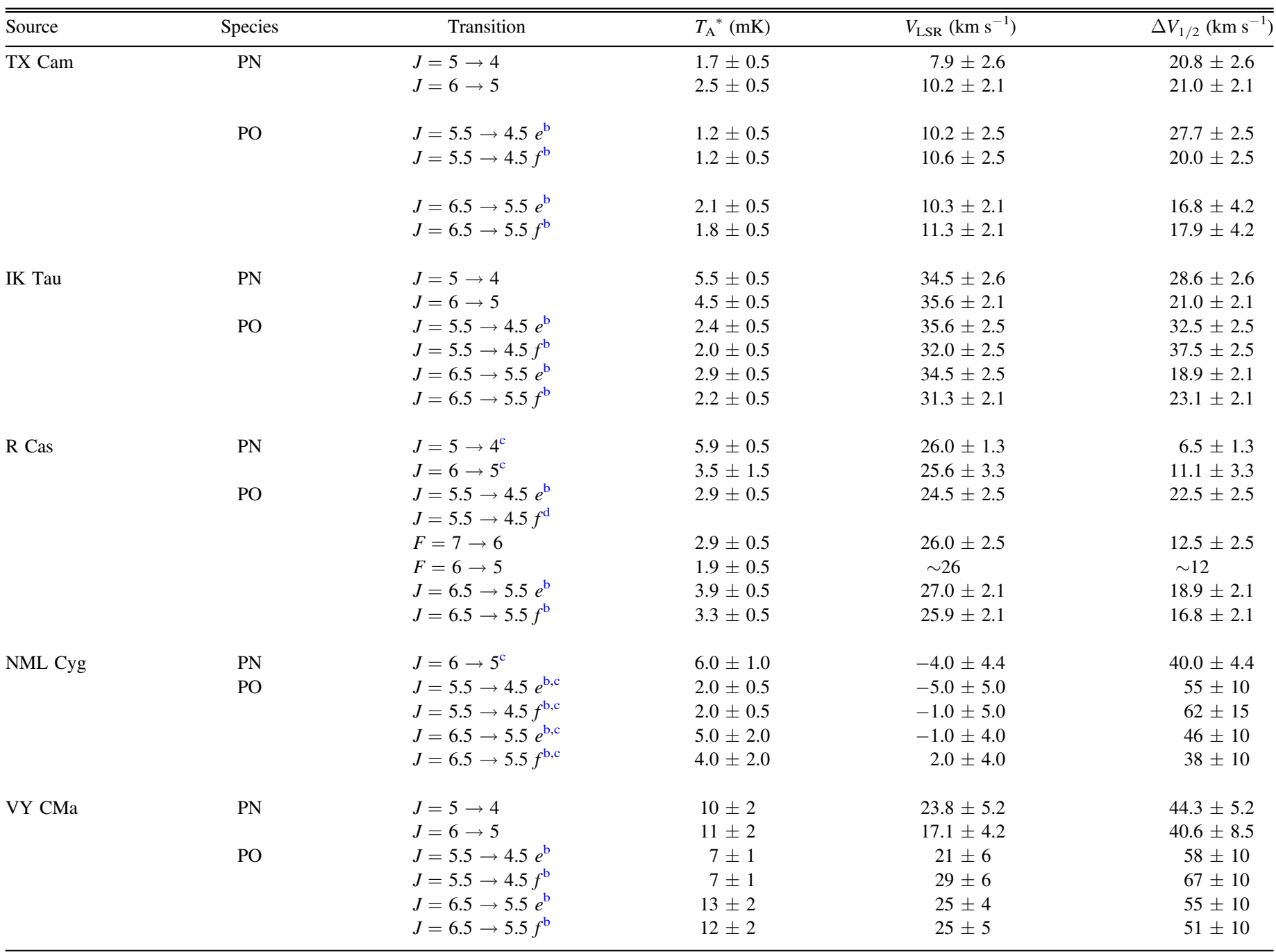

Notes.

${ }^{\text {a }}$ Measured with $2 \mathrm{MHz}$ resolution unless noted; VY CMa data from Tenenbaum et al. (2007, 2010).

b Blend of two hyperfine components; see Table 2 .

${ }^{\mathrm{c}}$ Measured with $1 \mathrm{MHz}$ resolution for PN; $4 \mathrm{MHz}$ resolution for PO.

${ }^{\mathrm{d}}$ Partially resolved hyperfine components; see Table 2.

various stars observed are given in Table 4, as well as the corresponding references. The density profile for the spherically symmetric outflows of all stars is described by the expression (Kemper et al. 2003; Adande et al. 2013):

$$
n_{\mathrm{H}_{2}}(r)=\frac{\dot{M}}{4 \pi V_{\exp }\left(1-\frac{R_{*}}{r}\right) r^{2}} .
$$

$V_{\text {exp }}$ is the average terminal expansion velocity of the stellar envelopes, as estimated from the measured line profiles, and $\dot{M}$ is the mass-loss rate (see Table 1). However, VY CMa also has highly directional, nonspherical outflows, which require an additional term in the density profile for the conical section depicting such flows (Adande et al. 2013),

$$
n_{\mathrm{H}_{2}}(r)=n_{0} \exp \left(-\left(\frac{r-r_{\text {peak }}}{r_{\text {outflow }}}\right)^{2}\right) .
$$

Here $r_{\text {peak }}$ is the radial distance at which the density reaches its maximum value of $n_{0}$, and $r_{\text {outflow }}$ is the distance where the density has dropped by a factor of $1 / \mathrm{e}$ from $r_{\text {peak }}$. It sets the radial extent of the outflow (see Adande et al. 2013 for further discussions). The $T_{\text {dust }}$ values were estimated based on the dust condensation radius of a given star and the stellar temperature (from the literature, see Tables 1 and 4), assuming the power law of Decin et al. (2006, see references therein).

The radial abundance profiles $f(r)$ for circumstellar envelopes typically are spherical or consist of shells, depending on the chemistry. The spherical distribution is modeled with the following profile, where $r_{\text {outer }}$ is the radial distance from the star at which the abundance has decreased by $1 / \mathrm{e}$, which sets the maximum extent of the sphere:

$$
f(r)=f_{0} \exp \left(-\left(\frac{r-r_{\text {inner }}}{r_{\text {outer }}}\right)^{2}\right) .
$$


Table 4

Modeling Parameters for Circumstellar Shells

\begin{tabular}{|c|c|c|c|c|c|c|}
\hline Source & $\begin{array}{c}V_{\exp } \\
\left(\mathrm{km} \mathrm{s}^{-1}\right)\end{array}$ & $\begin{array}{l}r_{\text {inner }} \\
(\mathrm{cm})\end{array}$ & $\begin{array}{c}T_{\text {dust }} \\
(\mathrm{K})\end{array}$ & $\begin{array}{c}r_{\text {profile }} \\
(\mathrm{cm})\end{array}$ & $\begin{array}{c}T_{\text {profile }} \\
(\mathrm{K})\end{array}$ & $\gamma$ \\
\hline TX Cam & 13.5 & $1.3 \times 10^{14 a}$ & 800 & $2.0 \times 10^{15}$ & 125 & 0.75 \\
\hline IK Tau & 16.0 & $2.0 \times 10^{14 b}$ & $726^{\mathrm{b}}$ & $1.8 \times 10^{14 \mathrm{c}}$ & 960 & 0.98 \\
\hline R Cas & 7.0 & $1.4 \times 10^{14 d}$ & $626^{\mathrm{c}}$ & $2.5 \times 10^{14 c}$ & 1050 & 0.91 \\
\hline VY CMa & 20.0 & $2.5 \times 10^{15 \mathrm{e}}$ & $670^{\mathrm{f}}$ & $1.0 \times 10^{16 \mathrm{~g}}$ & 230 & 0.62 \\
\hline NML Cyg & 24.0 & $5.0 \times 10^{15 h}$ & $580^{\mathrm{i}}$ & $1.0 \times 10^{16 \mathrm{~g}}$ & 270 & 0.50 \\
\hline
\end{tabular}

Notes.

${ }^{\mathrm{a}}$ Decin et al. (2010).

${ }^{b}$ Velilla Prieto et al. (2017).

c Maercker et al. (2016).

d Bauer \& Stencel (1994).

e Adande et al. (2013).

${ }^{f}$ Matsuura et al. (2014).

g Zubko et al. (2004).

h Ziurys et al. (2009).

${ }^{\mathrm{i}}$ Monnier et al. (1997).

For a shell, the profile is given by (e.g., Adande et al. 2013):

$$
f(r)=f_{0} \exp \left(-\left(\frac{r-r_{\text {shell }}}{r_{\text {outflow }}}\right)^{2}\right),
$$

where $r_{\text {shell }}$ is the distance at which the abundance reaches its maximum value of $f_{0}$; and $r_{\text {outflow }}$ is the distance from $r_{\text {shell }}$ at which the abundance has decreased by $1 / \mathrm{e}$ and defines the shell width. In this case, $r_{\text {outer }}=r_{\text {shell }}+r_{\text {outflow }}$, such that $r_{\text {outer }}$ is the distance from the star at the far side of the shell at which the abundance has fallen by $1 / \mathrm{e}$. The modeling in both cases begins at the distance from the star $r_{\text {inner, }}$ which was set to the dust condensation radius (see Table 4). Model parameters $f_{0}$, $r_{\text {outer }}$, and $r_{\text {shell }}$ are then varied to reproduce the observed line profiles.

To distinguish between source geometries, the data for PN and PO were first modeled with both spherical and shell distributions, and a chi-squared analysis was performed. For the spherical case, the $r_{\text {outer }}$ was typically varied from $40 R_{*}$ to $1500 R_{*}$, with the first increment of $60 R_{*}$ and then $100 R_{*}$ for successive iterations. For the shell geometry, the range $40 R_{*}$ to $1000 R_{*}$ was considered for both $r_{\text {shell }}$ and $r_{\text {outer }}$, with the same increments as the spherical model, holding $r_{\text {outer }} \geqslant r_{\text {shell }}$. In both analyses, the molecular abundance $f_{0}$ was varied between $10^{-9}$ and $10^{-7}$ in increments of 0.5 . All possible combinations of $r_{\text {outer }}, r_{\text {shell }}$ (when applicable), and $f_{0}$ were considered within the given ranges.

The analysis was first performed on the data for IK Tau, because two additional transitions $(J=2 \rightarrow 1$ and $J=3 \rightarrow 2$ lines) for PN were available from the IRAM $30 \mathrm{~m}$ data of De Beck et al. (2013), as well as the $\Omega=1 / 2, J=7 / 2 \rightarrow 5 / 2$ lambda doublets of PO. These supplemental single-dish data were included in the modeling. With the additional transitions to constrain the fit, the chi-squared analysis showed clearly that the spherical model was optimal for PN, as opposed to the shell, and vice versa for PO. Furthermore, the chi-squared values immediately demonstrated that using the $40 R_{*}$ radius assumed by De Beck et al. (2013) for IK Tau was far from optimal, and significantly larger values of $r_{\text {outer }}$ were necessary.

Chi-squared analyses for R Cas and NML Cyg gave identical results for the preferred molecular geometries as for IK Tau.
Based on these consistent outcomes, the PN data for all sources were modeled with a sphere, and the PO spectra with a shell, except the special case of VY CMa, which will be further discussed. The same range of radii and abundances were used as for IK Tau and optimized using chi-squared analyses. Note that $r_{\text {outer }}$ for NML Cyg was estimated from those of other stars, as only one transition was available for this source. Uncertainties in the respective source radii and abundances were assigned on the basis of the chi-squared values. These values showed clear minima; a deviation from the minimum value between $10 \%$ and $30 \%$ defined the assigned uncertainties. There were few redundancies, except in the case of PN for NML Cyg, as mentioned.

VY CMa is a special case, as the envelope of this source exhibits multiple asymmetric outflows (e.g., Humphreys et al. 2005; Smith et al. 2009; Ziurys et al. 2009), which complicate molecular profiles. Although sensitive single-dish spectra of $\mathrm{SO}_{2}$ and $\mathrm{SO}$ exhibit at least five different velocity components (e.g., Tenenbaum et al. 2010; Adande et al. 2013), the emission from PO and PN were too weak to warrant such a detailed analysis. Therefore, fits were attempted for both molecules using a simplistic model consisting of three velocity components: a spherically symmetric wind, one collimated blueshifted outflow, and a collimated redshifted flow. As described by Adande et al. (2013), the spherical outflow is likely the vestige of past symmetric mass loss, while the blueand redshifted outflows are possible remnants of large bursts of mass ejection (e.g., Humphreys et al. 2005). The position angle and vertex angle for the two directional outflows are equivalent to those listed for "Blue Outflow" and "Red Outflow 1" of Adande et al. (2013). The abundances in the collimated outflows were modeled with $r_{\text {peak }}$ and $r_{\text {outer }}$ using Equation (5), with $r_{\text {peak }}$ substituted for $r_{\text {shell }}$. A similar range of trial values and chi-squared optimization were performed for the VY CMa data. It was found that the PN spectra were best modeled with all three velocity components; for PO, the spherical flow sufficed, and no improvement in the chi-squared value resulted from including the additional velocity components.

Although VY CMa is a complicated source, the ESCAPADE model worked quite well in reproducing single-dish observations of $\mathrm{SO}_{2}$ and $\mathrm{SO}$ - both which had sharp, asymmetric 
Table 5

Abundances and Distributions for PN and PO in O-rich Stars

\begin{tabular}{|c|c|c|c|c|c|c|}
\hline \multirow[b]{2}{*}{ Source } & \multicolumn{3}{|c|}{ PN } & \multicolumn{3}{|c|}{$\mathrm{PO}$} \\
\hline & $f_{0}\left(10^{-8}\right)$ & $r_{\text {outer }}\left(R_{*}\right)$ & $r_{\text {peak }}{ }^{\mathrm{a}}\left(R_{*}\right)$ & $f_{0}\left(10^{-8}\right)$ & $r_{\text {shell }}\left(R_{*}\right)$ & $r_{\text {outer }}^{\mathrm{b}}\left(R_{*}\right)$ \\
\hline TX Cam & $1.0 \pm 0.3$ & $500 \pm 100$ & $\cdots$ & $5.5 \pm 2.5$ & $100 \pm 50$ & $200 \pm 50$ \\
\hline IK Tau & $1.0 \pm 0.2$ & $900 \pm 200$ & $\ldots$ & $4.5 \pm 2.5$ & $200 \pm 50$ & $300 \pm 50$ \\
\hline NML Cyg & $0.3 \pm 0.1$ & $500 \pm 300$ & $\cdots$ & $7 \pm 3$ & $40 \pm 20$ & $50 \pm 20$ \\
\hline \multicolumn{7}{|l|}{ VY CMa } \\
\hline Spherical Wind & $0.7 \pm 0.3$ & $200 \pm 50$ & $\ldots$ & $5 \pm 3$ & $\ldots$ & $50 \pm 20$ \\
\hline
\end{tabular}

Notes.

${ }^{\mathrm{a}}$ Collimated flows only (see the text).

${ }^{\mathrm{b}} r_{\text {outer }}=r_{\text {shell }}+r_{\text {outflow }}$ (see the text).

blue- and redshifted features that simply cannot be reproduced by a spherical geometry (see Adande et al. 2013). The modeling not only successfully fit numerous, complex spectra (27 transitions in the case of $\mathrm{SO}_{2}$, covering a wide energy range), but also produced spatial distributions in good agreement with those of $\mathrm{SO}_{2}$ from interferometer maps $(\mathrm{Fu}$ et al. 2012), as well as with the collimated outflows traced in IR dust emission (Humphreys et al. 2005; Smith et al. 2009).

Results of the modeling are presented in Table 5 and in Figure 3. In the figure, radial abundance plots for both molecules (PN: green or black; PO: orange or gray-scale) are shown for the AGB shells (left panels) and the supergiants (right panels), as derived from the modeling. For VY CMa, the three outflows in PN are shown on the graph: spherical (solid line), red (asymmetric dash) and blue (symmetric dash), as described. The kinetic temperature function is also plotted (purple or gray-scale), with the corresponding legend on the right. In Table 5, the peak fractional abundances $f_{0}$ and relevant radial distances $\left(r_{\text {shell }}, r_{\text {peak, }}\right.$ and $\left.r_{\text {outer }}\right)$ are summarized for both molecules, as calculated from the models and depicted in Figure 3.

\section{Discussion}

\subsection{New Circumstellar Detections of PO and PN}

This study has resulted in the discovery of three new circumstellar sources of PO and PN: TX Cam, R Cas, and NML Cyg. Phosphorus monoxide has now been found in five circumstellar shells, three from AGB stars and two associated with supergiants. All these stars are oxygen-rich. PN has now been identified in seven circumstellar envelopes. Two such objects are carbon-rich (IRC+10216 and CRL 2688: Milam et al. 2008), while the other five are O-rich. These findings clearly suggest that phosphorus-bearing molecules are common constituents in circumstellar gas and are likely to be found toward other evolved stars. Other phosphorus-containing species, such as HPO and $\mathrm{PH}_{3}$, might be present as well in O-rich envelopes. As discussed in the next section, if the abundance of phosphorus in these envelopes is near to the cosmic value, then as much as $\sim 20 \%$ of this element is in the form of gas-phase molecules.

These results also present somewhat of a quandary for models of dust grain formation. Thermodynamic calculations of grain condensation in both $\mathrm{C}$ and O-rich circumstellar gas suggest that phosphorus, being a refractory element, becomes incorporated into the iron/nickel mineral schreibersite (e.g., Lodders \& Fegley 1999). This prediction keeps with the condensation temperature of phosphorus, which is $\sim 1150 \mathrm{~K}$ (e.g., Savage \& Sembach 1996). Note that those of silicon and magnesium are close in value-about $1300 \mathrm{~K}$ in both cases. It could be that the condensation process is simply not as efficient as thought, or that shock waves may be disrupting grain formation. All three AGB stars in this study are Mira variables, and hence have pulsating atmospheres that expand and contract, which invariably leads to shock propagation in the stellar envelope (e.g., Cherchneff 2012). The supergiants also create shocked material with their sporadic mass loss (e.g., Adande et al. 2013). Such shock waves can disrupt chemical equilibrium and any associated condensation processes (Gobrecht et al. 2016).

\subsection{Abundances and Circumstellar Distributions of $P O$ and $P N$}

As summarized in Figure 3 and Table 5, PO and PN in all three AGB stars have similar abundances and distributions. The peak fractional abundance of $\mathrm{PN}$ falls in the range $f_{0}$ $[\mathrm{PN}] /\left[\mathrm{H}_{2}\right] \sim 1-2 \times 10^{-8}$, with $r_{\text {outer }} \sim 500-900 R_{*}$. In the case of phosphorus monoxide, $f_{0}[\mathrm{PO}] /\left[\mathrm{H}_{2}\right] \sim 0.5-1 \times 10^{-7}$, with the shell distributions maximizing at $r_{\text {shell }} \sim 100-200 R_{*}$. The abundance of PN remains high in the outer shell, well after that of PO has significantly decreased. In all three objects, PO is more abundant than PN by a factor of 5, considering peak values, which is consistent with the oxygen-rich nature of these envelopes. Both species appear to be most prevalent in the envelope of $\mathrm{R}$ Cas, a relatively unstudied source.

In their study of IK Tau, De Beck et al. (2013) determined higher abundances for both $\mathrm{PO}$ and $\mathrm{PN}$ than was determined here. They found $f[\mathrm{PN}] /\left[\mathrm{H}_{2}\right] \sim 3 \times 10^{-7}$ and $f[\mathrm{PO}] /\left[\mathrm{H}_{2}\right] \sim$ $0.5-6 \times 10^{-7}$. These values were established with the assumption of a $\sim 40 R_{*}$ source size, which was based solely on what was found for VY CMa. Our chi-squared analysis, which included IRAM $30 \mathrm{~m}$ results, are simply not consistent with such a confined distribution, as discussed previously. Our abundances were also derived from a radiative transfer analysis, which for circumstellar shells is likely to be more robust than those derived from a rotational diagram. Despite these differences, the lower end of the abundance range quoted by De Beck et al. for PO matches our value. Also note that if the higher value of $6 \times 10^{-7}$ from De Beck et al. (2013) is considered for PO, then the total abundance of both molecules 

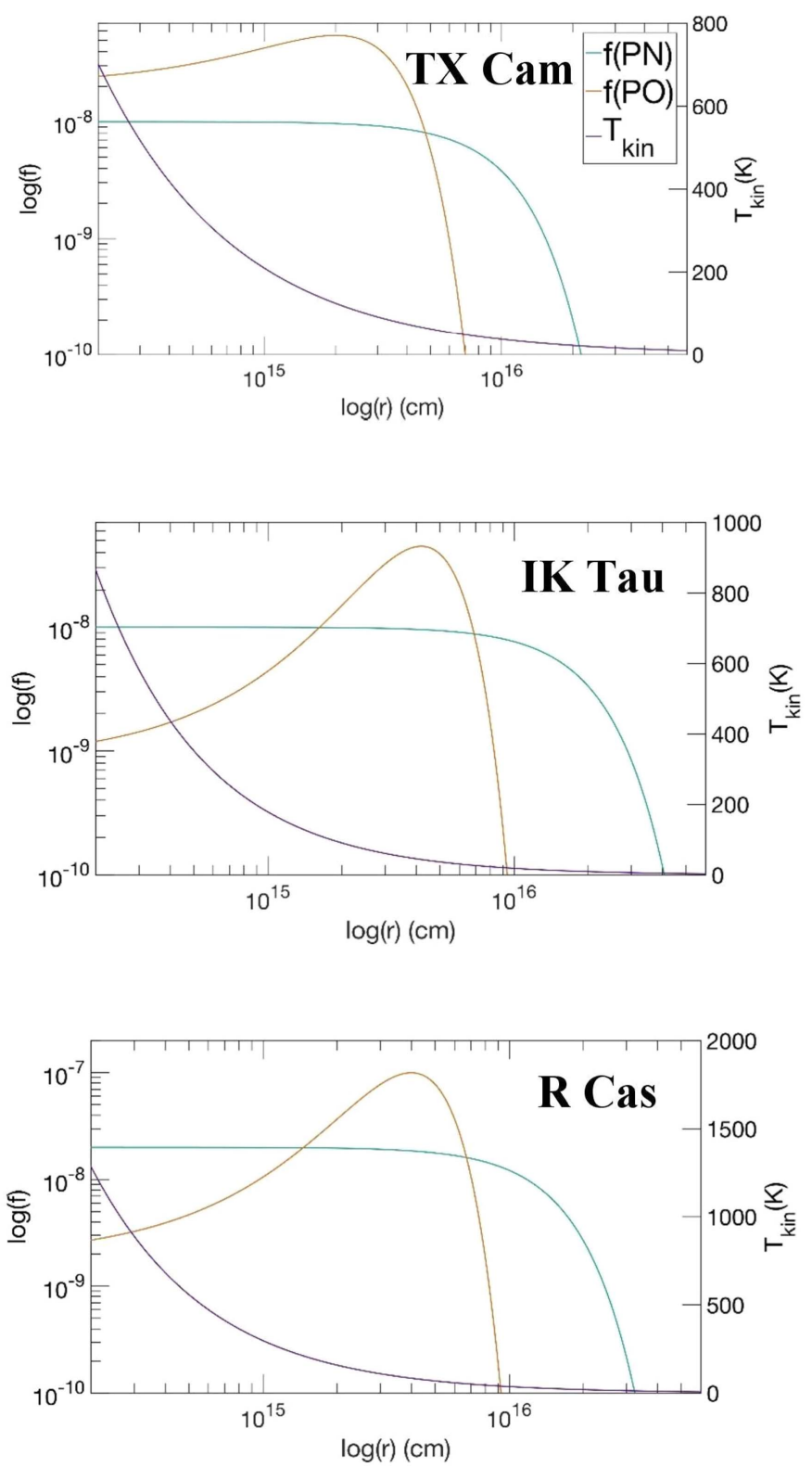
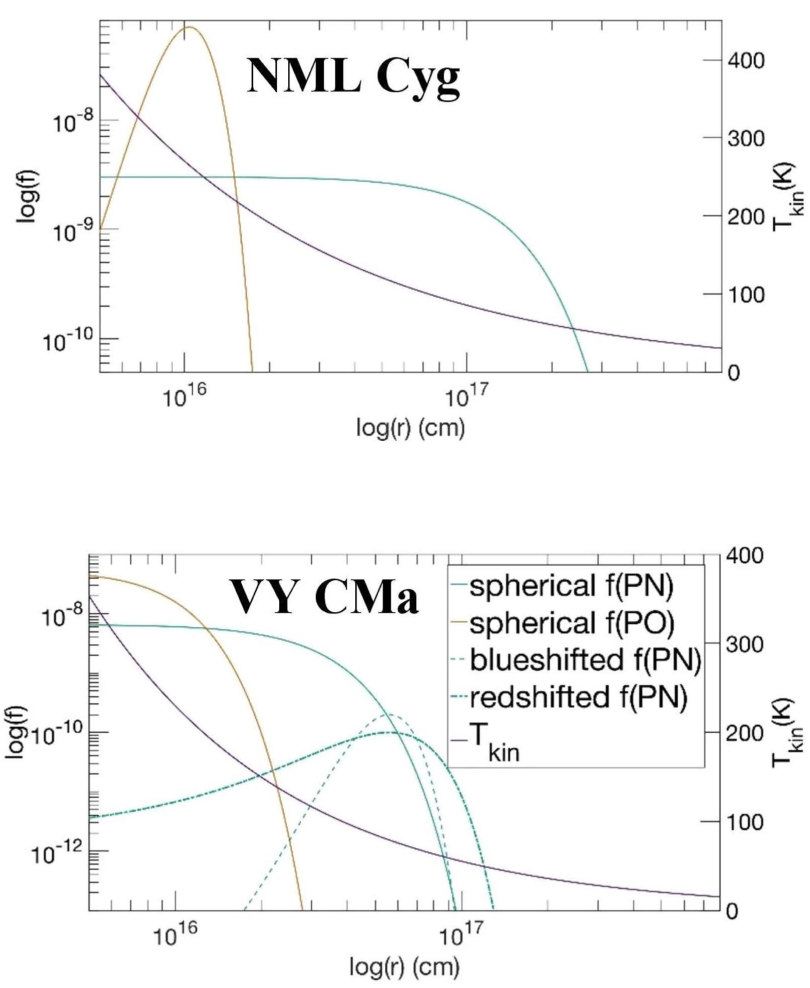

Figure 3. Radial abundance plots ( $\log f$ vs. $\log r$ (in $\mathrm{cm}$ )) for PN (orange) and PO (green), determined from ESCAPADE modeling. Kinetic temperature as a function of $\log r$, based on the temperature profile parameters given in Table 4, is also plotted (purple), with the corresponding scale on the right $y$-axis. Left: TX Cam, IK Tau, and R Cas. Right: NML Cyg and VY CMa. For VY CMa, three outflows are shown for PN: spherical (solid line), red (asymmetric dash) and blue (symmetric dash). The molecular distributions suggest that PN is a parent molecule formed near the stellar photosphere, while PO is created further out in the envelope.

would be $9 \times 10^{-7}$. This value is higher than the cosmic abundance of phosphorus. It would suggest that AGB stars are producing this element and that it is all contained in PN and PO. This scenario is unlikely.

The abundances of PN in the envelopes of the two supergiants are somewhat lower (factor of 2-7) than those on the AGB stars. The outflows from VY CMa, and likely for NML Cyg as well, are more energetic with multiple, nonspherical winds (e.g., Ziurys et al. 2009), resulting in a different chemical environment. The radiative transfer analysis suggests that $\mathrm{PN}$ is less prevalent in NML Cyg than any other object studied here, with $f_{0}[\mathrm{PN}] /\left[\mathrm{H}_{2}\right] \sim 3 \times 10^{-9}$. However, only one transition of the molecule was measured toward this source, so the abundance is not as well constrained. In VY $\mathrm{CMa}$, the overall abundance of PN is slightly less than in the AGB stars $\left(f_{0} \sim 7 \times 10^{-9}\right)$. The molecule is most prevalent in the spherical flow, as opposed to the highly directional winds, by about a factor of 70 . The dominance of the spherical flow is apparent in the line profile; the prominent red- and blueshifted features are barely visible, and the bulk of the emission is at the LSR velocity of the star (e.g., Tenenbaum et al. 2010). The abundance of $\mathrm{PO}$ in both objects is roughly comparable, with $f_{0} \sim(5-7) \times 10^{-8}$, which is similar to those in AGB stars, with the spherical wind in VY CMa again being the major carrier. In the shell of NML Cyg, PO is more abundant that PN by a factor of $\sim 20$, unlike the AGB stars; in VY CMa, PN is roughly a factor of 7 less prevalent than PO. De Beck et al. (2013) also found PO to be about a factor of 7 more abundant than PN in this source.

The distribution of both molecules in NML Cyg is similar to that in the AGB stars, with the PN abundance achieving a maximum at a radial distance of a few hundred stellar radii and 
PO exhibiting a maximum closer to the star. The modeling in this case is likely an oversimplification, because NML Cyg exhibits molecular-line profiles that obviously have multiple velocity components (D. R. Schmidt et al. 2018, in preparation). For VY CMa, the two species appear to be located within $\sim 50-200 R_{*}$ of the star for the spherical flow, which is reasonably consistent with the interferometer results of De Beck et al. (2013). For the red- and blueshifted flows, PN has abundances that reach maximum values at roughly $500 R_{*}$ and $600 R_{*}$. These radial distances correspond to the positions of the various arcs observed in the infrared (South Arc, Arc2, NW Arc, etc.), some which appear to be associated with the red- and blueshifted molecular outflows (e.g., Smith et al. 2009; Adande et al. 2013).

\subsection{Implications for Phosphorus Chemistry}

There are few models available in the literature that focus on phosphorus chemistry in O-rich stars. One exception is the work of MacKay \& Charnley (2001). Their model suggests that PO is formed in the outer circumstellar envelope where photodissociation begins to occur. Phosphorus monoxide is formed from either atomic P or PS through the reactions:

$$
\begin{gathered}
\mathrm{P}+\mathrm{OH} \rightarrow \mathrm{PO}+\mathrm{H} . \\
\mathrm{PS}+\mathrm{OH} \rightarrow \mathrm{PO}+\mathrm{SH} .
\end{gathered}
$$

Both reactions are thought to be exothermic. These reactions become most effective at a stellar radius near $2 \times 10^{16} \mathrm{~cm}$-which is roughly comparable to where the PO abundance reached its maximum for all five stars (see Figure 3). However, the maximum amount of PO produced by the MacKay \& Charnley (2001) model was $f \sim 10^{-10}$, or factors of 100-1000 lower than the observed values.

A more recent study by Gobrecht et al. (2016) concerns the chemistry in IK Tau, within $10 R_{*}$ of the star. These authors perform calculations of molecular abundances in the gas phase under LTE conditions, and then allow a shock wave to propagate through the material. The ensuing "shock" abundances are then computed. They also consider grain "cluster" formation as well. In their model, $\mathrm{PO}$ and $\mathrm{PN}$ are both considered, but not phosphorus-bearing grains. In contrast to the MacKay \& Charnley (2001) predictions, these authors compute a high LTE abundance of PO of $\sim 10^{-7}$; this value quickly drops to $(2-3) \times 10^{-10}$ as the shock impacts the chemistry. On the other hand, Gobrecht et al. (2016) predict that PN has a lower concentration at LTE $\left(\sim 4 \times 10^{-10}\right)$, which then rapidly increases in the shocked gas to $\sim 6 \times 10^{-7}$ by $6 R_{*}$. PN is created in the shock from PO, via the process:

$$
\mathrm{PO}+\mathrm{N} \rightarrow \mathrm{PN}+\mathrm{O} \text {. }
$$

$\mathrm{PO}$ is likewise formed from the reaction of Equation (7), or from destruction of $\mathrm{PN}$ :

$$
\mathrm{PN}+\mathrm{OH} \rightarrow \mathrm{PO}+\mathrm{NH} .
$$

The predicted abundance for PO from this model at the maximum radial distance considered $\left(6 R_{*}\right)$ is several orders of magnitude too low in comparison to that derived from the observations, either here or from De Beck et al. (2013). Calculations were not carried out beyond $10 R_{*}$, so a further assessment is not possible. The abundance computed for PN, again at $6 R_{*}$, is higher by about a factor of 30-60 than those determined in this work. The differences between these model predictions and the observations would suggest that shocks are not the main chemical pathway to producing PO. A non-shock formation mechanism, perhaps photochemically induced, could generate PO further out in the envelope. PN, in contrast, may be a product of shock chemistry near the stellar photosphere; it would then be transported through the shell with decreasing abundance with radial distance.

The observed line profiles give some remote clues into the chemistry of PO and PN. The spectral shapes of both molecules are very similar to those found in other species in the AGB stars (e.g., Ziurys et al. 2009) within the uncertainties, with typical linewidths and LSR velocities. PO and PN are present in gas that has reached the terminal outflow velocity in each star and are present well beyond the inner envelope. For the case of VY CMa, the broad linewidths for PN indicate that this molecule is present in the collimated blue- and redshifted outflows (see Ziurys et al. 2009), and therefore may be formed by shocks in these regions (see Adande et al. 2013). The envelope of NML Cyg is not sufficiently well understood to draw any concrete conclusions from the line profiles, but it likely also has a complex outflow structure and possible shocked regions. Complicated line profiles have been observed in various molecules toward this source as well (D. R. Schmidt et al. 2018, in preparation). In any case, a combination of chemical processes may be necessary to synthesize phosphorus-bearing molecules. Additional modeling of phosphorus chemistry, especially for oxygen-rich evolved stars, would be highly desirable.

\section{Conclusions}

This work clearly shows that phosphorus-containing molecules are common in circumstellar shells of O-rich stars, including those on the AGB and also supergiants. Overall, the formation of PO is apparently favored over that of PN in oxygen-rich circumstellar material. Both molecules also typically have significant abundances at radial distances of a few hundred stellar radii, at least in the AGB stars. Furthermore, this study indicates that there are major gas-phase carriers of phosphorus, and condensation onto grains may not be as important for this element as was previously thought. Studies of additional envelopes would be enlightening.

We thank the staff of ARO for making these observations possible. This research was supported by NSF grants AST1515568 and AST-1140030. The SMT is operated by the Arizona Radio Observatory (ARO), with partial support through the NSF University Radio Observatories (URO) program (AST-1140030). The work was also supported by NASA under Agreement No. NNX15AD94G issued through the Science Mission Directorate interdivisional initiative Nexus for Exoplanet System Science (NExSS).

\section{References}

Adande, G. R. 2013, PhD thesis, Univ. Arizona

Adande, G. R., Edwards, J. L., \& Ziurys, L. M. 2013, ApJ, 778, 22

Agúndez, M., Cernicharo, J., Decin, L., Encrenaz, P., \& Teyssier, D. 2014, ApJL, 790, L27

Agúndez, M., Cernicharo, J., \& Guélin, M, J. 2007, ApJL, 662, L91

Agúndez, M., Cernicharo, J., Pardo, J. R., Guélin, M., \& Phillips, T. G. 2008, A\&A, 485, L33

Asplund, M., Grevesse, N., Sauval, A. J., \& Scott, P. 2009, ARA\&A, 47, 481 Bauer, W. H., \& Stencel, R. E. 1994, AJ, 107, 2233

Caffau, E., Bonifacio, P., Faraggiana, R., \& Steffen, M. 2011, A\&A, 532, A98 Cescutti, G., Matteucci, F., Caffau, E., \& François, P. 2012, A\&A, 540, A33 Cherchneff, I. 2012, A\&A, 545, A12 
Choi, Y. K., Hirota, T., Honma, M., et al. 2008, PASJ, 60, 1007

De Beck, E., Decin, L., de Koter, A., et al. 2010, A\&A, 523, A18

De Beck, E., Kamiñski, T., Patel, N. A., et al. 2013, A\&A, 558, A132

Decin, L., De Beck, E., Brünken, S., et al. 2010, A\&A, 516, A69

Decin, L., Hony, S., de Koter, A., et al. 2006, A\&A, 456, 549

Duari, D., Cherchneff, I., \& Willacy, K. 1999, A\&A, 341, L47

Dubernet, M. L., Alexander, M. H., Ba, Y. A., et al. 2013, A\&A, 553, A50

Fontani, F., Rivilla, V. M., Caselli, P., Vasyunin, A., \& Palau, A. 2016, ApJL, 822, L30

Fu, R., Moullet, A., Patel, N. A., et al. 2012, ApJ, 746, 42

Gobrecht, D., Cherchneff, I., Sarangi, A., Plane, J. M. C., \& Bromley, S. T. 2016, A\&A, 585, A6

Halfen, D. T., Clouthier, D. J., \& Ziurys, L. M. 2008, ApJL, 677, L101

Humphreys, R. M., Davidson, K., Ruch, G., \& Wallerstein, G. 2005, AJ, 129, 492

Jacobsen, H. R., Thanathibodee, T., Frebel, A., et al. 2014, ApJL, 796, L24

Justtanont, K., de Jong, T., Helmich, F. P., et al. 1996, A\&A, 315, L217

Kemper, F., Stark, R., Justtanont, K., et al. 2003, A\&A, 407, 609

Kłos, J., Lique, F., \& Alexander, M. H. 2008, CPL, 455, 1

Lebouteiller, V., Kuassivi, V., \& Ferlet, R. 2006, in ASP Conf. Ser. 348 Astrophysics in the Far Ultraviolet: Five years of Discovery with FUSE, ed. G. Sonneborn, H. W. Moos, \& B.-G. Anderson (Victoria, BC: ASP), 480

Lefloch, B., Vastel, C., Viti, S., et al. 2016, MNRAS, 462, 3937

Lodders, K., \& Fegley, B. 1999, in IAU 191, Asymptotic Giant Branch Stars, ed. T. Le Bertre, A. Lèbre, \& C. Waelkens (Montpellier: IAU), 279

Maciá, E. 2005, Chem. Soc. Rev., 34, 691

MacKay, D. D. S., \& Charnley, S. B. 2001, MNRAS, 325, 545

Maercker, M., Danilovich, T., Olofsson, H., et al. 2016, A\&A, 591, A44

Maercker, M., Schöier, F. L., Olofsson, H., Bergman, P., \& Ramstedt, S. 2008, A\&A, 479, 779
Markwick, A. J., \& Millar, T. J. 2000, A\&A, 359, 1162

Massey, P., \& Thompson, A. B. 1991, AJ, 101, 1408

Matsuura, M., Yates, J. A., Barlow, M. J., et al. 2014, MNRAS, 437, 532

McSween, H. Y., \& Huss, G. R. 2010, in Cosmochemistry, ed. H. Y. McSween \& G. R. Huss (Cambridge: Cambridge Univ. Press), 120

Milam, S. N., Halfen, D. T., Tenenbaum, E. D., et al. 2008, ApJ, 684, 618

Monnier, J. D., Bester, M., Danchi, W. C., et al. 1997, ApJ, 481, 420

Olofsson, H., Lindqvist, M., Nyman, L-A., \& Winnberg, A. 1998, A\&A, 329, 1059

Pasek, M. A., \& Lauretta, D. S. 2005, AsBio, 5, 515

Savage, B. D., \& Sembach, K. R. 1996, ARA\&A, 34, 279

Smith, N., Hinkle, K. H., \& Ryde, N. 2009, AJ, 137, 3558

Tenenbaum, E. D., Dodd, J. L., Milam, S. N., Woolf, N. J., \& Ziurys, L. M. 2010, ApJS, 190, 348

Tenenbaum, E. D., Woolf, N. J., \& Ziurys, L. M. 2007, ApJL, 666, L29

Tenenbaum, E. D., \& Ziurys, L. M. 2008, ApJL, 680, L121

Tobola, R., Kłos, J., Lique, F., Chałasiñski, G., \& Alexander, M. H. 2007 A\&A, 468, 1123

Turner, B. E., Tsuji, T., Bally, J., Guelin, M., \& Cernicharo, J. 1990, ApJ, 365, 569

Velilla Prieto, L., Sánchez Contreras, C., Cernicharo, J., et al. 2017, A\&A, 597, A25

Ziurys, L. M. 1987, ApJL, 321, L81

Ziurys, L. M., Milam, S. N., Apponi, A. J., \& Woolf, N. J. 2007, Natur, 447, 1094

Ziurys, L. M., Tenenbaum, E. D., Pulliam, R. L., Woolf, N. J., \& Milam, S. N. 2009, ApJ, 695, 1604

Zubko, V., Li, D., Lim, T., Feuchtgruber, H., \& Harwit, M. 2004, ApJ, 610, 427 